\title{
The associations of acceptance with quality of life and mental health following spinal cord injury: a systematic review
}

\author{
Anders Aaby $\mathbb{1}^{1,2,3} \cdot$ Sophie Lykkegaard Ravn ${ }^{1,2,3} \cdot$ Helge Kasch $\mathbb{1}^{4} \cdot$ Tonny Elmose Andersen ${ }^{1,2}$
}

Received: 5 July 2019 / Revised: 23 October 2019 / Accepted: 24 October 2019 / Published online: 12 November 2019

(c) The Author(s), under exclusive licence to International Spinal Cord Society 2019

\begin{abstract}
Study design Systematic review.

Objectives To identify, critically appraise, and synthesize research findings on the associations between acceptance, quality of life (QOL), and mental health outcomes in individuals living with spinal cord injury (SCI).

Methods Five databases (PubMed, PsycINFO, Embase, Web of Science, and Scopus) were systematically searched. Studies were included if they provided findings on the association between acceptance and QOL, mental health outcomes, or both in an SCI population aged 16 years or older. Only peer-reviewed original quantitative and qualitative studies were included. Screening, quality assessment, and data extraction were conducted independently by two researchers. Findings were tabulated and synthesized by outcome.

Results Forty-one studies were included. Greater acceptance was consistently associated with greater global and psychological QOL, life satisfaction, sense of well-being, mental health, and with lower levels of depression and anxiety. Inconsistent evidence was found with regards to social QOL and post-traumatic stress disorder. Acceptance was generally not associated with adjustment outcomes further than 2 years into the future. Study quality of the quantitative studies was mostly fair $(n=17)$ followed by good $(n=13)$, and poor $(n=9)$.

Conclusion Health-care professionals may regard acceptance as a psychological resource they can aim to support in improving QOL and mental health following SCI. A range of methodological and conceptual limitations were present in the research. Future studies should prioritize longitudinal designs, consider dyadic effects, explore subjective meaning(s) of acceptance, and investigate the effectiveness of therapeutic approaches that stimulate the acceptance process.
\end{abstract}

\section{Introduction}

A spinal cord injury (SCI) is a severe and chronic condition that is often accompanied by paralysis, numerous secondary

Supplementary information The online version of this article (https:// doi.org/10.1038/s41393-019-0379-9) contains supplementary material, which is available to authorized users.

Anders Aaby

aaaby@health.sdu.dk

1 InCoRE, Department of Psychology, University of Southern Denmark, Odense, Denmark

2 ThRIVE, Department of Psychology, University of Southern Denmark, Odense, Denmark

3 The Specialized Hospital for Polio and Accident Victims, Roedovre, Denmark

4 Spinal Cord Injury Center of Western Denmark, Viborg, Denmark complications, functional impairments, and persistent pain [1]. This type of injury causes profound and irretrievable changes in many aspects of life [2] and has repeatedly been described as one of the most devastating injuries a person can experience $[3,4]$. Individuals with SCI are faced with a myriad of daily challenges, including disruptions in work [5], family and social relationships [6], and community participation [7]. Furthermore, some individuals with SCI experience reduced satisfaction with life [8], poor social participation [9], and up to $30 \%$ report clinical levels of depression and anxiety at some point following their injury [10]. Hence, psychological distress is common in SCI and has been shown to persist up to 10 years or more post injury [11].

The presence and severity of negative psychological outcomes are influenced by many factors, including acceptance of the injury [12-14]. Acceptance is defined as perceiving the injury as an integral part of life going forward and a reevaluation of life values [15], and thus 
provides a coping strategy that is particularly relevant in unalterable situations [16]. Empirical studies show that greater acceptance is associated with greater quality of life (QOL) [17] and with lower levels of depression [13], anxiety [18], and post-traumatic stress disorder (PTSD) [19]. However, these empirical studies often focus more generally on psychological factors such as the global construct of coping, where acceptance is only incidentally included [13, 17-19]. This all-encompassing approach has some benefits, but it complicates the matter of getting a detailed overview of the specific role of acceptance in the adjustment process. This complication was highlighted in a previous systematic review from 2012 on psychological factors and QOL following SCI [4]. In this review acceptance was associated with lower levels of depression and anxiety as well as higher satisfaction with life, but due to the large-scale nature of their review, the authors recommended that future research provide more detailed insights into the factors that were consistently related to favorable adjustment outcomes [4]. However, a systematic review on the associations between acceptance, QOL, and mental health outcomes does not currently exist.

The aim of the present systematic review was to identify, critically appraise, and synthesize relevant research findings to answer the research question of how acceptance is associated with QOL and mental health outcomes in individuals living with SCI.

\section{Theoretical foundation}

Over time, acceptance has been conceptualized in a variety of ways. Initially, stage-theory approaches of adjustment to SCI and other disabilities conceptualized acceptance as the result of successful progression through predefined stages such as denial, anger, bargaining, and depression [20]. However, these theories relied on clinical observations rather than empirical studies [21] and are generally not supported today [20]. Another cornerstone that continues to be a major influence is the seminal work of Wright [22]. Wright proposed a theory of disability acceptance as an adjustment of a person's value system. In this framework, acceptance entails that the perceived losses resulting from the disability do not devalue existing abilities or the person as a whole $[22,23]$. While the theory of disability acceptance is still relevant today, in recent years most of the empirical researchers have turned their attention to the stress and coping literature [20]. The transactional model of stress and coping is based on the premise that psychological distress is caused by appraising a situation as exceeding the personal and situational resources needed to manage that situation [24]. In this framework, acceptance is proposed to reduce the discrepancy between what one prioritizes in life and what one is capable of doing, thus reducing psychological distress [25]. One of the newest developments in this theoretical framework is the Spinal Cord Injury Adjustment Model (SCIAM) [20]. This is a dynamic, multivariate model of the adjustment process following SCI, which proposes that biological, psychological, and social factors all influence adjustment outcomes via appraisals and coping strategies.

In empirical studies, acceptance is generally conceptualized and measured in relation to two aspects: "accepting reality" and "value-change". The accepting reality aspect focuses on an acknowledgement of reality as it is in the present moment, whereas the value-change aspect focuses on changing one's perspective and learning to appreciate new aspects of life [15].

\section{Methods}

This systematic review was conducted and reported in accordance with the PRISIMA checklist [26], and a review protocol was registered in PROSPERO on January 29, 2019 (ID: CRD42019118644).

\section{Search strategy}

A systematic literature search identified peer-reviewed publications exploring an association between acceptance and either QOL or mental health outcomes in individuals with SCI. On September 3, 2018 five databases (PubMed, PsycINFO, Embase, Web of Science, and Scopus) were systematically searched for eligible studies. An update search was performed on September 5, 2019 to identify studies published in the preceding year. All results and figures in this report include data from the updated search. The preliminary work consisted of pilot searches to ensure exhaustiveness of search terms and to identify appropriate Subject Headings and Medical Subject Headings (MeSH). These preliminary searches indicated that a search strategy focused on synonyms and related terms of SCI (e.g., spinal cord lesion) and acceptance (e.g., coping) would yield a manageable total sum of records. Therefore, no further specifiers were added. A final search algorithm was developed using Boolean logic operators ("AND" and "OR"). Search terms available as a subject heading or a MeSH term were searched both as such without explosion and as free text in title/abstract/keywords or similar. No restrictions were put on publication date. The search algorithm was performed as uniformly as possible across databases. The research librarians at University of Southern Denmark provided feedback on the search algorithm. The full electronic search algorithm in PsycINFO is provided in Supplementary Appendix 1 as an example. 


\section{Eligibility criteria}

Inclusion and exclusion criteria were developed prior to screening. Peer-reviewed original quantitative and qualitative research papers written in English, Danish, Norwegian, or Swedish were included, while reviews, doctoral dissertations, protocols, conference abstracts, and editorials were excluded. To be included, studies had to explore the association between acceptance and either QOL, mental health outcomes, or both among individuals with SCI aged 16 or older. Note that the $Q O L$ construct also comprised measures of life satisfaction and well-being, while mental health included measures of depression, anxiety, and PTSD. Studies focusing on narrower constructs such as hopelessness, functional independence, and social isolation, were not included. Furthermore, acceptance was conceptualized as either an acknowledgement of the permanence of the injury, as a reevaluation of life values, or a combination of the two. Studies were selected based on their use of standardized measures of acceptance, QOL, and mental health that have demonstrated adequate validity, such as the Spinal Cord Lesion-related Coping Strategies Questionnaire (SCL-CSQ) [15], the Coping Orientations to Problems Experienced (COPE) Inventory [16], the acceptance of disability scale (ADS) [27], World Health Organization Quality of Life Questionnaire [28], and Patient Health Questionnaire-9 [29]. Readers are encouraged to visit the SCIRE website at www.scireproject.com for an easy-access description of many of the scales used in the included studies. Lastly, studies were excluded if they only dealt with inborn spinal cord conditions or disease entities, such as spinal cord infarction, myelitis, or spinal stenosis or if they only involved second analyses of a sample, but not adding new results to this review.

\section{Screening}

All results from the searches were imported into EndNote, and duplicate records were identified and removed prior to screening. Next, all unique records were transferred to the online tool Covidence [30], and screened in title and abstracts according to the eligibility criteria (step 1). Records that could not be excluded based on title or abstract were full-text screened (step 2). At this step, reasons for exclusion were registered. The screening process was carried out by two raters independently, and disagreements between raters were discussed at each step. A third researcher was consulted when necessary.

\section{Quality assessment}

The U.S. National Institutes of Health's Quality Assessment Tool for Observational Cohort and Cross-Sectional
Studies (OCCSS) was used to appraise the quantitative studies [31] and the Critical Appraisal Skills Programme (CASP) was used to appraise the qualitative studies [32]. The OCCSS includes 14 criteria and has a final global rating scale of poor, fair, and good. Studies that were longitudinal or statistically controlled for confounders, or both, were given a quality rating of good unless there were other considerable methodological issues. Studies with a cross-sectional design that lacked any statistical control for confounders were given a quality rating of fair, unless there were considerable methodological issues (e.g., missing eligibility criteria or unclear sample population), in which case they were rated poor. The CASP includes ten questions that support a critical appraisal of qualitative evidence. It does not produce a final rating. Quality assessments were carried out independently by two raters. Disagreements were discussed, and a third researcher was consulted when necessary. Studies were not excluded based on their quality rating, and the studies were not weighted according to quality in the synthesis. Further, no analysis of quality across studies was conducted. Instead, study quality across studies (such as potential publication bias) was discussed at a general level.

\section{Data extraction and synthesis}

Data extraction included sample characteristics (i.e., sample size, age, gender, type of injury, and time since injury), study country and setting, study design and time of measurement, the measure of acceptance, measure(s) of adjustment, and key findings of the study. Only findings relevant to address the research question were extracted. This information was tabulated. Data extraction was conducted independently by two raters. Disagreements were resolved by checking the original paper. The authors of the original paper were contacted in three instances to clarify ambiguous results. These results are marked with an asterisk in Table 1 containing the extracted data.

The data synthesis was done separately for cross sectional and longitudinal studies, and both followed the same three-step procedure: (1) direction and significance of correlations between acceptance and outcome, (2) the association between acceptance and an outcome in multiple regression analyses with control for potential confounding variables, and (3) any associations derived from other statistical methodologies (e.g., cluster analysis, latent growth mixture modeling, or qualitative findings). The synthesis was organized according to the psychological correlates examined in the individual studies. These were (1) global and specific domains of QOL, (2) well-being, (3) satisfaction with life, (4) mental health, (5) depression, (6) anxiety, and (7) PTSD. 


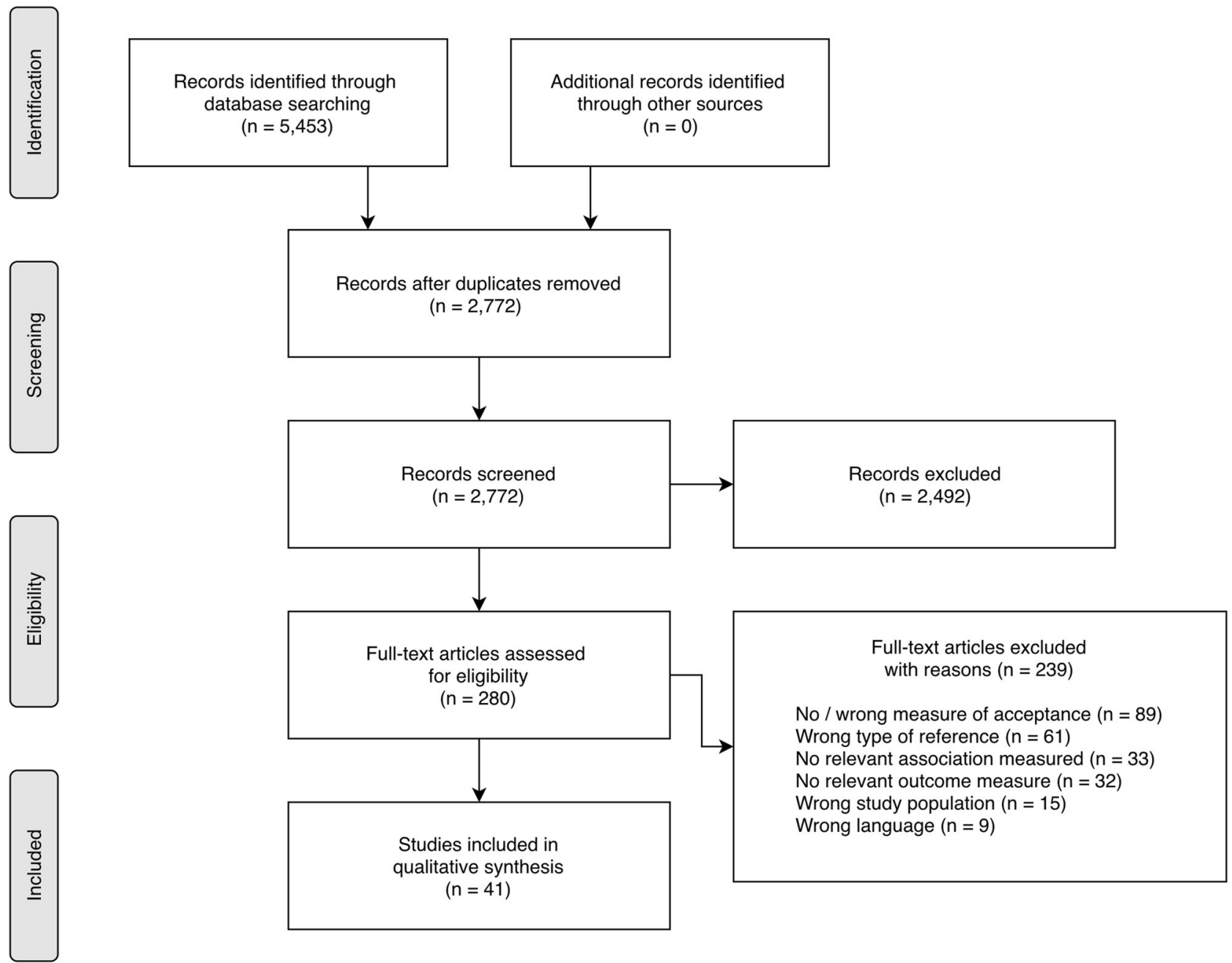

Fig. 1 PRISMA Flowchart of the systematic search and screening process, including reasons for exclusion. Final search was performed on September 5, 2019

\section{Results}

\section{Systematic search results}

The search retrieved 2772 unique records, of which 2492 were excluded in title and abstract screening, and 239 excluded in full-text screening. Thus, 41 studies were included in this review (see Fig.1). There were 39 quantitative studies and two qualitative ones.

\section{Study characteristics of the included studies}

Most studies were cross-sectional $(n=30)$ with sample sizes between 12 and 511. All studies had a majority of male participants, and the mean age ranged from 30 to 63 years. Mean time since injury varied extensively from 6 weeks post injury to 24 years post injury. The majority of studies were conducted in western developed countries. Six scales were used to measure acceptance (see Table 1) with the three most used being the SCL-CSQ [15] $(n=23)$, the COPE [16] $(n=6)$, and the ADS [27] $(n=6)$. The included studies explored associations between acceptance and global and specific domains of QOL $(n=19)$, satisfaction with life $(n=4)$, well-being $(n=4)$, mental health $(n=1)$, depression $(n=26)$, anxiety $(n=18)$, and PTSD $(n=2)$. Many studies explored more than one of these associations.

\section{Study quality}

Of the 39 quantitative studies, nine studies received a study quality rating of poor, 17 a rating of fair, and 13 a rating of good. Recurrent quality issues were a lack of longitudinal study designs, a lack of statistical control for confounders, poorly defined study populations, and low participation rates. A summary of the ratings and main issues of each study is provided in Supplementary Appendix 2. Quality assessment of the qualitative studies revealed appropriate research designs and data collection for both studies, but an inappropriate 


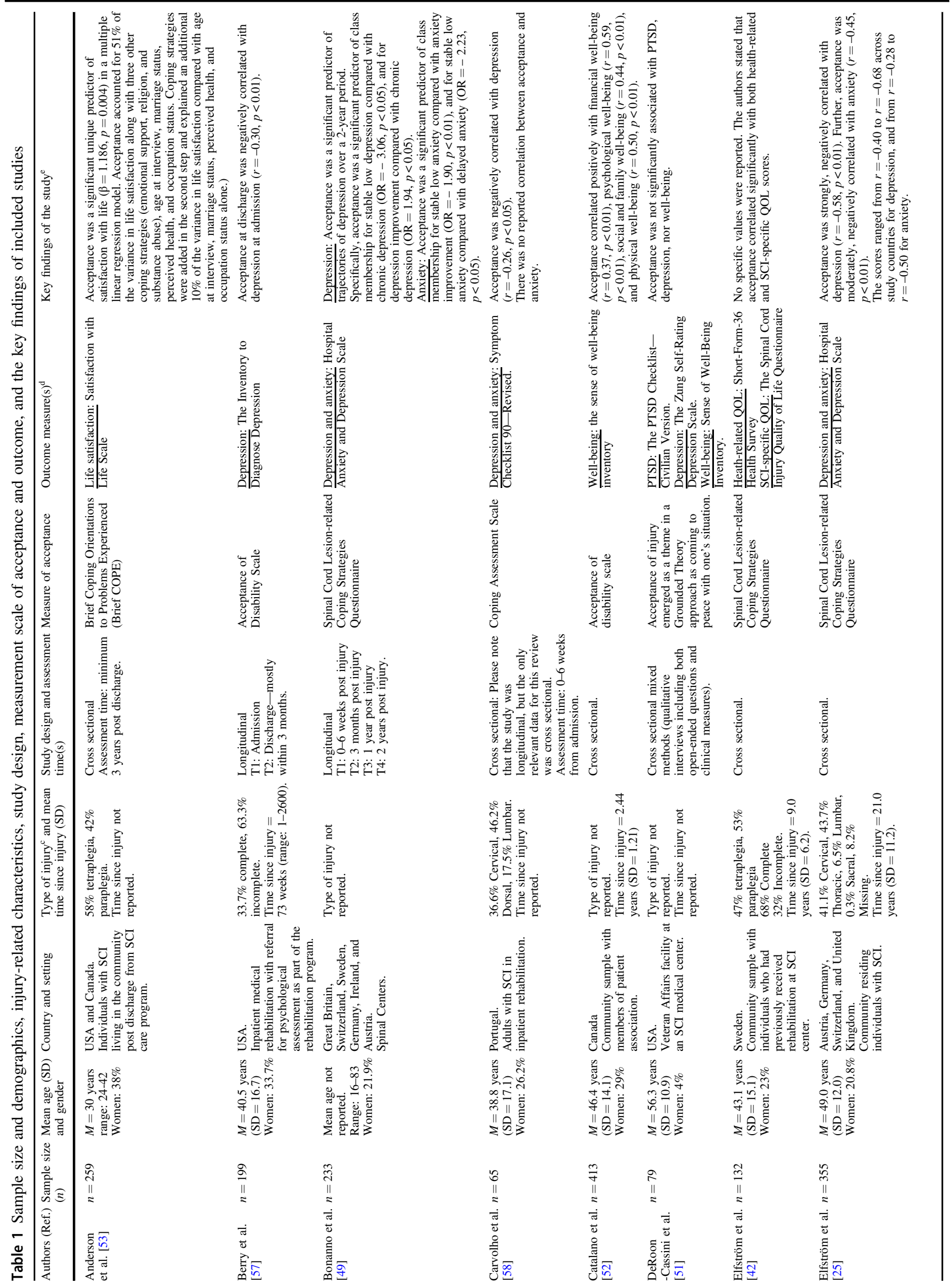




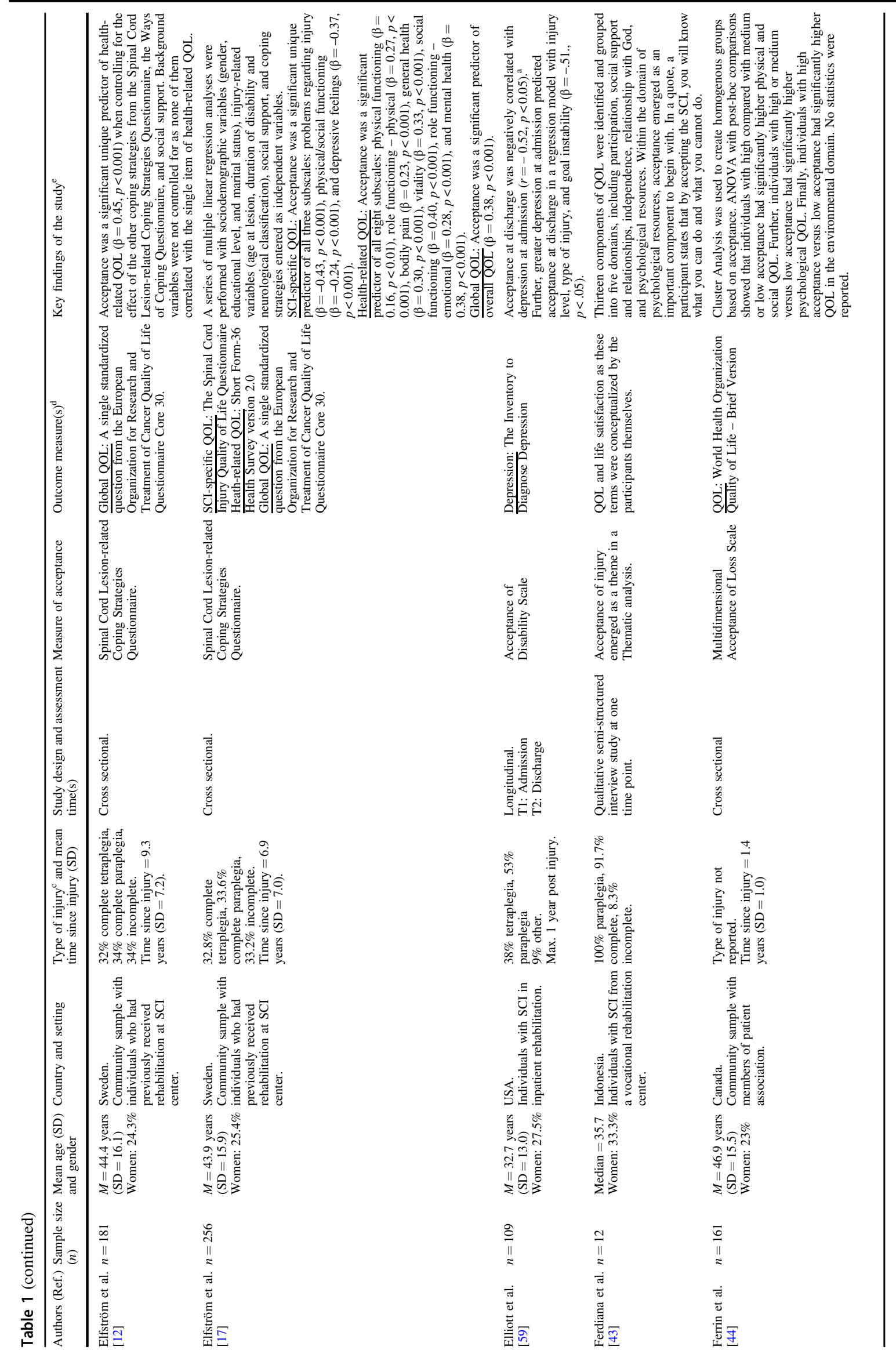




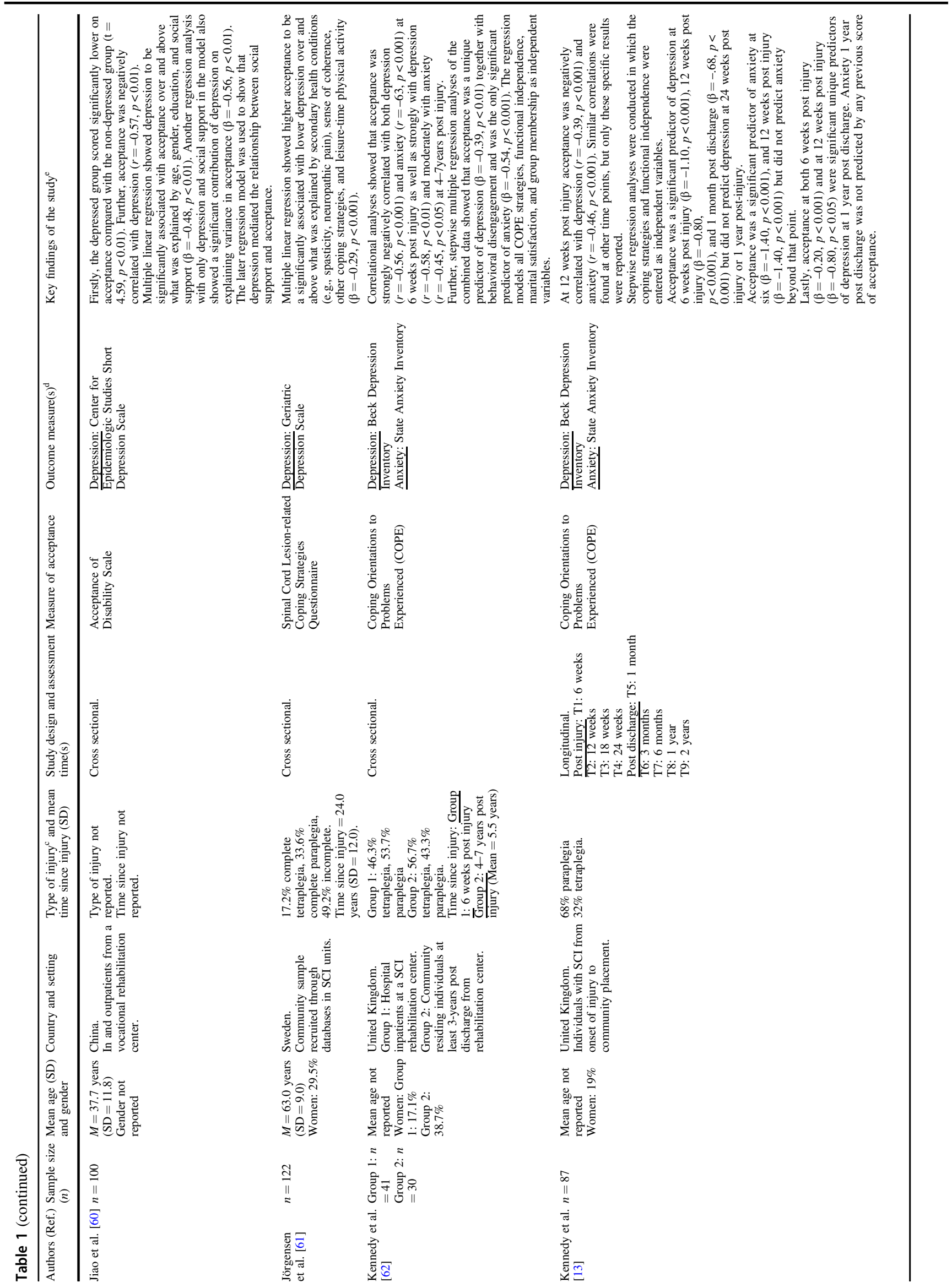




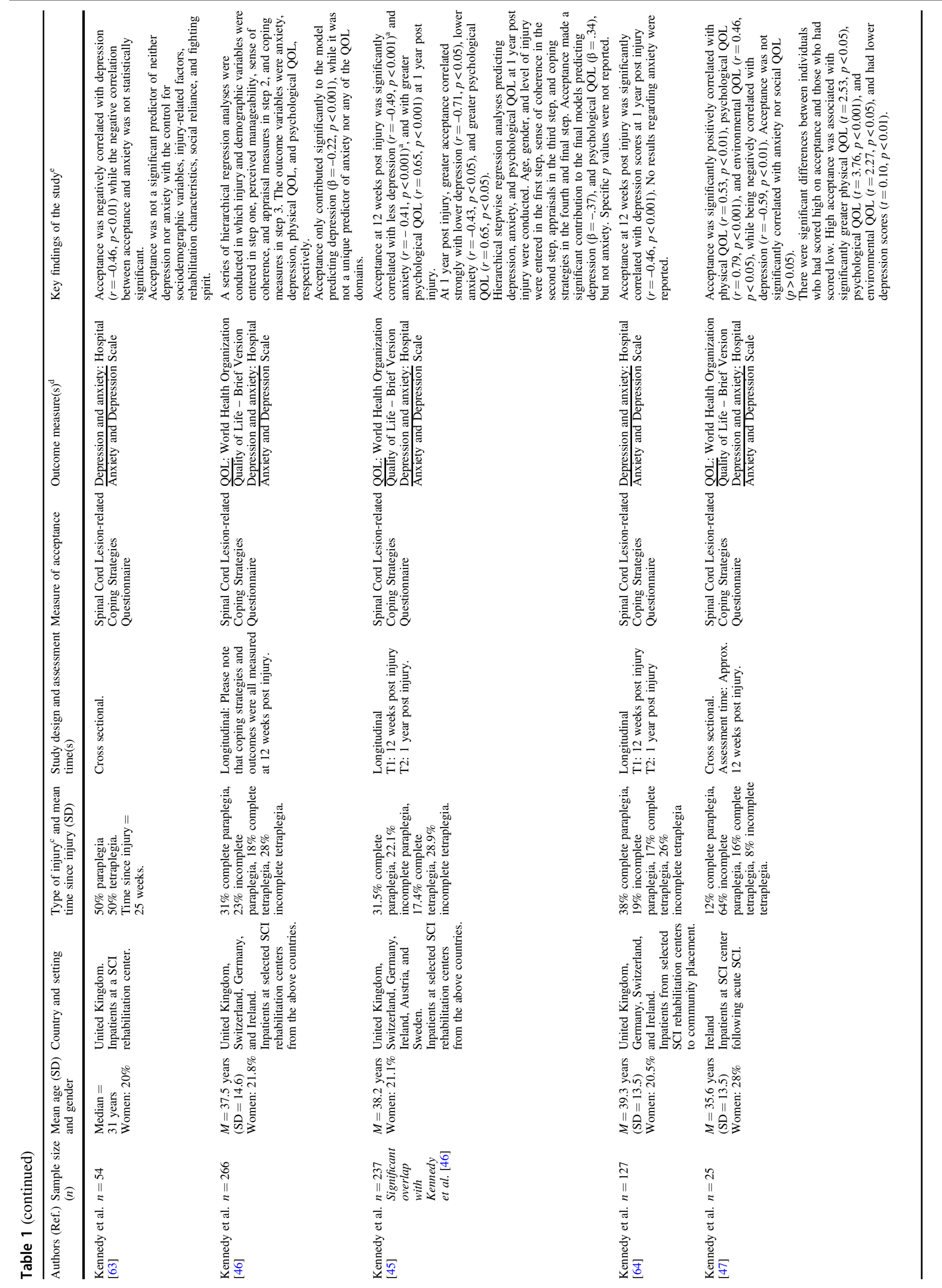




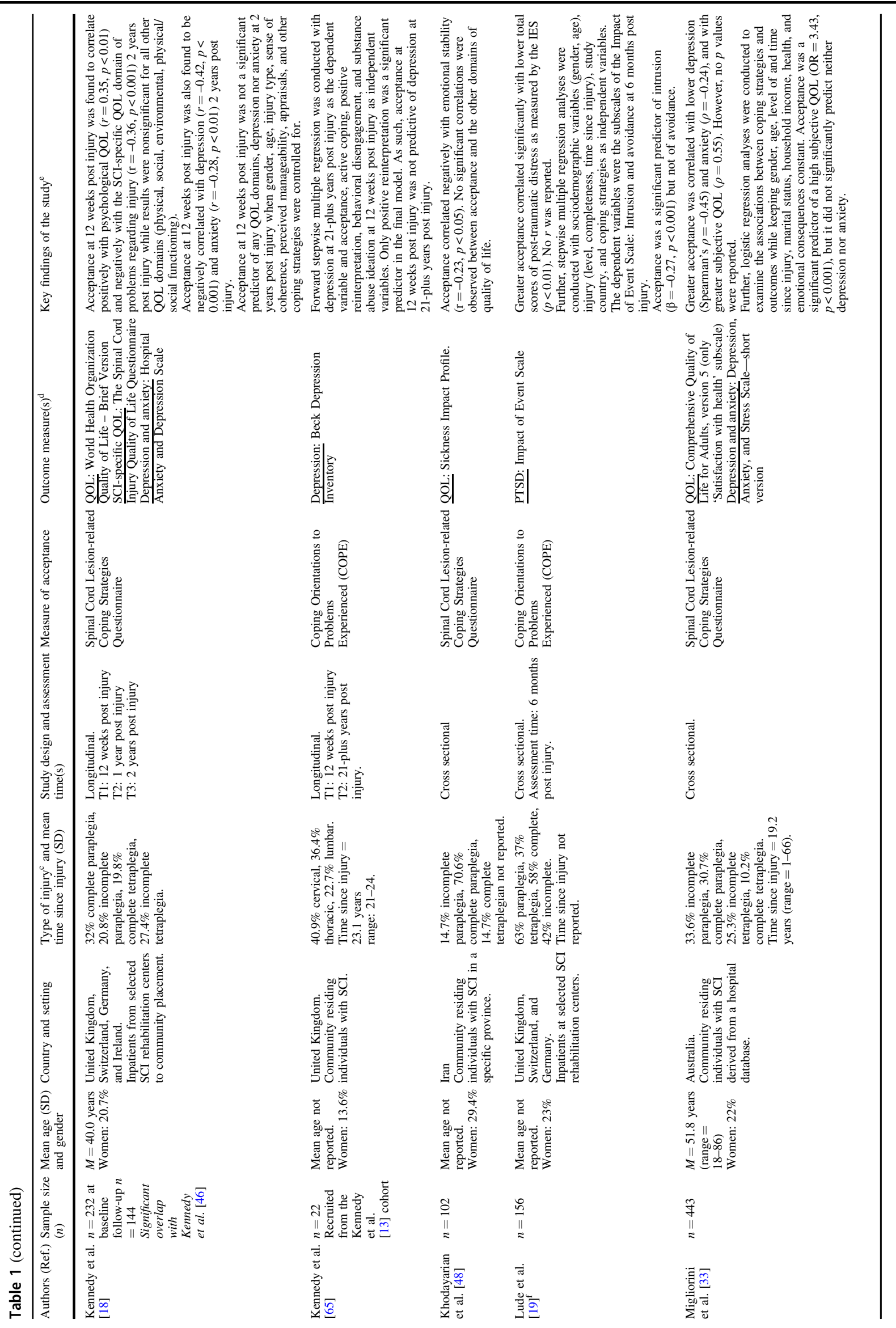




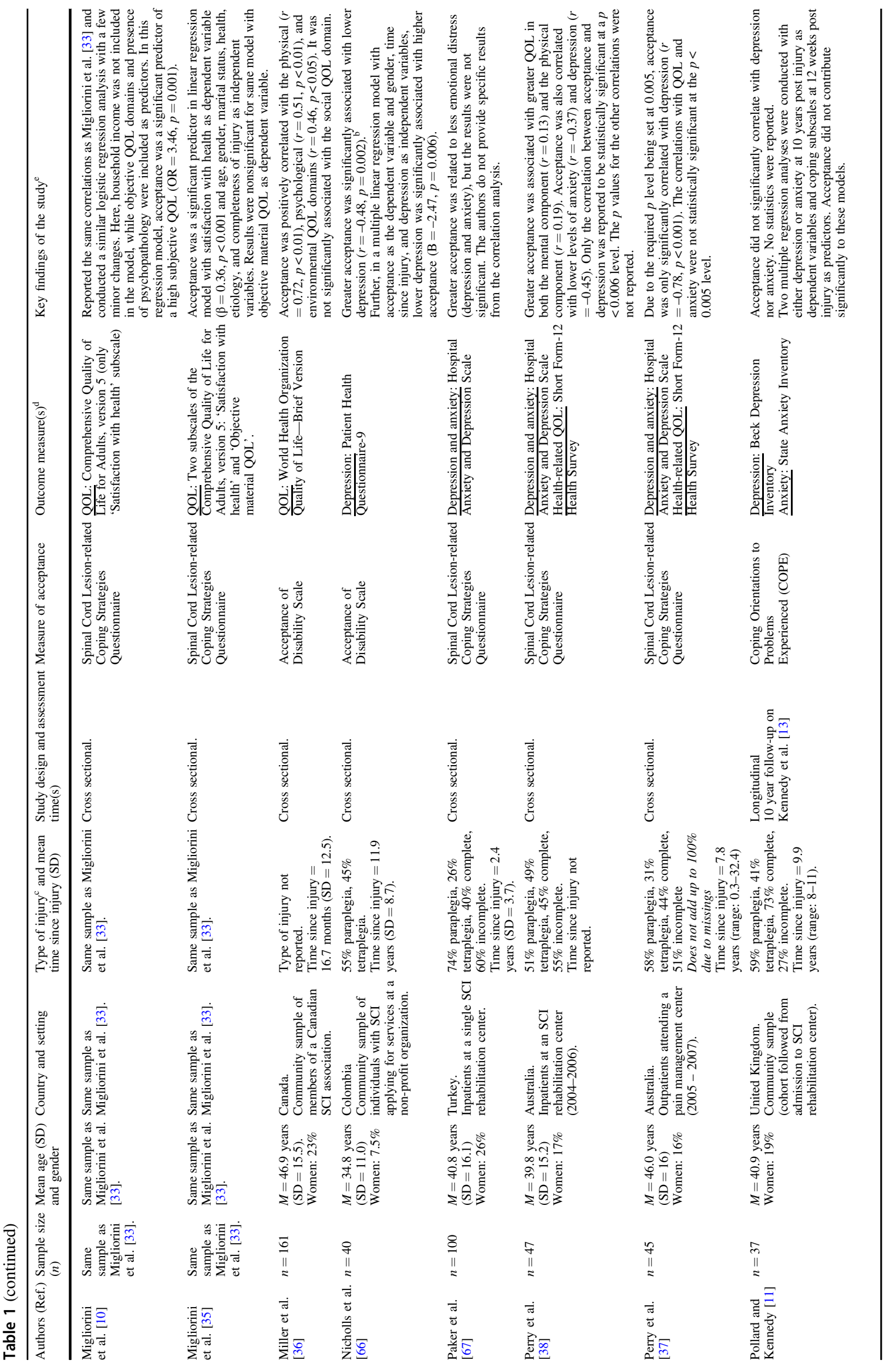




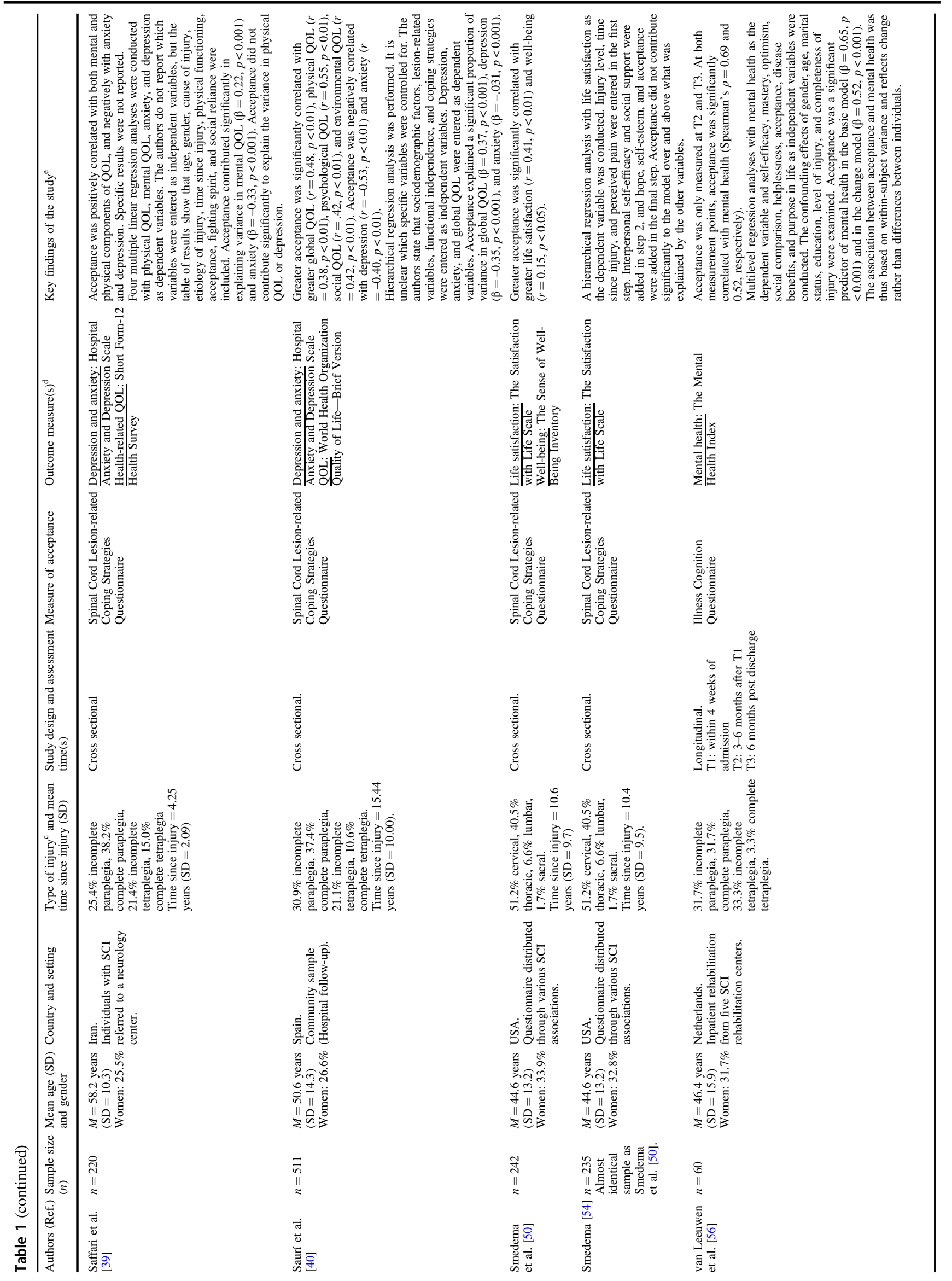




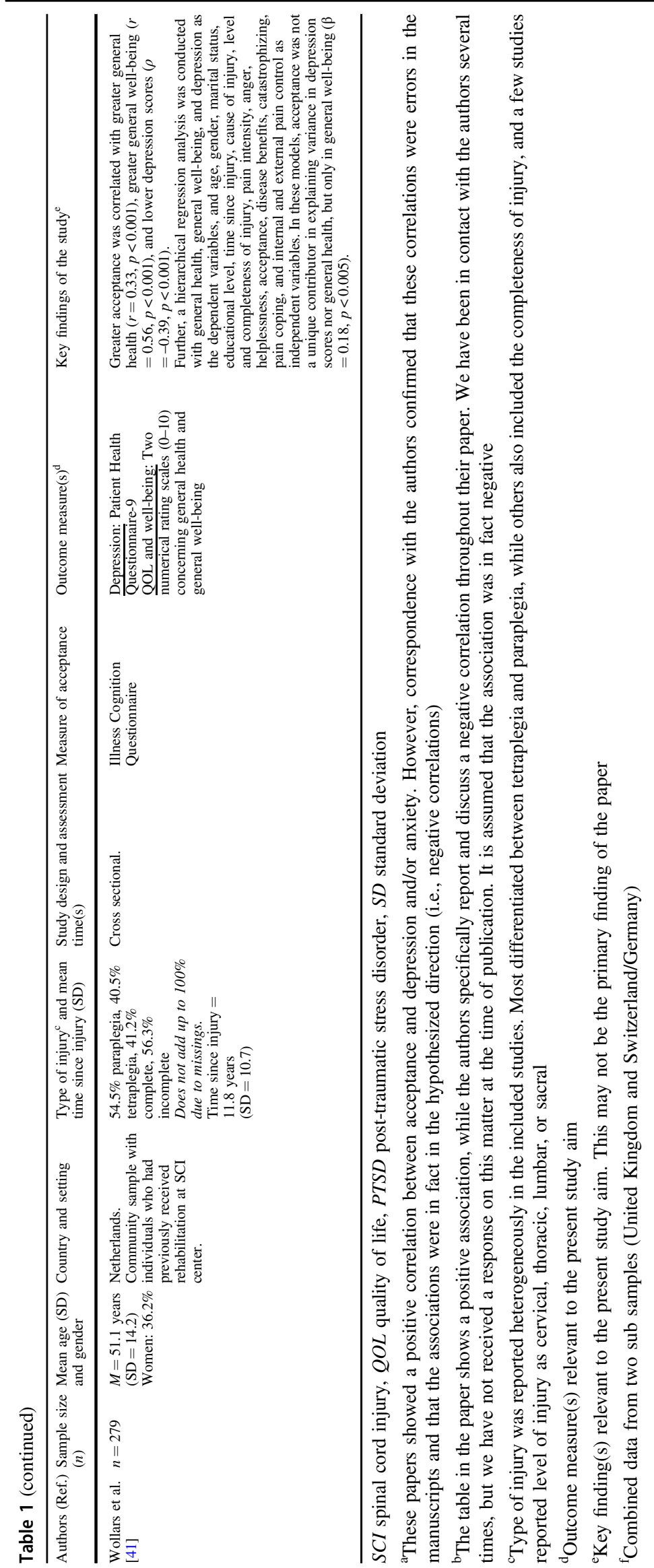


recruitment strategy and inadequate discussion of findings in one.

\section{Data synthesis}

\section{Global and specific domains of quality of life}

The association between acceptance and QOL was explored in 19 studies [12, 17, 18, 33-48]. In cross-sectional studies, greater acceptance was consistently associated with greater QOL [12, 17, 33-42, 44, 47]. Furthermore, acceptance was a significant predictor of global QOL even when sociodemographic factors (e.g., gender, age, marital status), injury-related variables (e.g., cause and type of injury, time since injury), and the other coping strategies of the SCLCSQ (i.e., fighting spirit and social reliance) [12, 17] were controlled for. Moreover, acceptance was associated with a greater likelihood of reporting greater global QOL [33, 34]. A single qualitative study found acceptance to be a component of QOL, and was grouped with maintaining goals and motivation into the domain of psychological resources [43]. No longitudinal studies focused on global QOL.

Acceptance was also consistently correlated with greater psychological QOL in all cross-sectional studies [36, 39, 40, 44, 47]. Individuals who were moderate or high in acceptance reported significantly greater psychological QOL compared with individuals who were low in acceptance [44]. In longitudinal studies acceptance at 6 to 12 weeks post injury was significantly correlated with psychological QOL 1 and 2 years post injury [45, 49]. Furthermore, acceptance was a significant predictor of psychological QOL at 1 year post injury even when the effect of sociodemographic factors, injury-related variables, sense of coherence, cognitive appraisals, and additional coping strategies were controlled for [45]. However, acceptance was not a significant predictor of psychological QOL at 12 weeks [46] and 2 years post injury [18] when sociodemographic factors, injury-related variables, perceived manageability, sense of coherence, cognitive appraisals, and coping strategies were controlled for.

In contrast to global and psychological QOL, the association between acceptance and social QOL was inconsistent. One cross-sectional study found a significant correlation [40], and acceptance was further a significant predictor of social QOL with control for sociodemographic factors, injury-related variables, social support, and coping strategies [17]. Conversely, three other cross-sectional studies and a 2-year follow-up study reported non-significant correlations [18, 36, 47, 48].

\section{Well-being}

The association between acceptance and well-being was investigated in four cross-sectional studies [41, 50-52] and was consistently associated with a greater sense of wellbeing $[41,50,52]$ with only one reporting a non-significant result [51]. Specifically, acceptance was positively correlated with general well-being [41, 50], social and family well-being [52], and psychological well-being [52]. Acceptance was also a predictor of general well-being with control for the effect of sociodemographic factors, injuryrelated variables, pain intensity, anger, helplessness, disease benefits, catastrophizing, pain coping, and internal and external pain control [41]. No longitudinal studies explored this association.

\section{Satisfaction with life}

Associations between acceptance and satisfaction with life were explored in four cross-sectional studies $[35,50,53,54]$. Their findings consistently indicated correlations between acceptance and greater satisfaction with life [50, 54]. Acceptance was a significant predictor of satisfaction with life [53] and satisfaction with health [35] even when sociodemographic factors, injury-related variables, and coping strategies were controlled for. However, acceptance was not a significant predictor of life satisfaction when injury-related variables, pain, self-efficacy, social support, hope, and self-esteem were controlled for [54]. No longitudinal studies explored this association.

\section{Mental health}

One longitudinal study explored the association between acceptance and mental health as a global construct [55]. Acceptance was significantly correlated with greater mental health both during and after discharge from rehabilitation [56]. Further, multilevel regression analyses showed that changes in acceptance over time were associated with improvements in mental health even when controlling for potential confounding variables (see Table 1). This indicated that the association was based on within-subject variance rather than differences between individuals.

\section{Depression}

The associations between acceptance and depression were investigated in 26 studies $[11,13,18,25,33,37-41,45-$ 47, 49, 51, 57-67]. Greater acceptance was associated with lower depression in most cross-sectional studies $[13,25,33,37-41,47,57,58,60-63,66]$, with only two studies reporting nonsignificant correlations [51, 67]. When sociodemographic factors, injury-related variables, and additional psychosocial variables were controlled for, acceptance was a significant predictor of depression in four studies [13, 40, 61, 62], while three studies showed nonsignificant results $[13,39,63]$. One longitudinal study 
conducted a series of regression analyses on cross-sectional data and reported that acceptance was associated with depression scores at six and 12 weeks post injury as well as 1 month post discharge from a rehabilitation center [13]. This was not the case at 24 weeks post injury nor 1 year post discharge [13]. The longitudinal data generally showed a similar tendency. Acceptance measured within 6 to 12 weeks post injury correlated significantly with depression at one [45] and 2 years post injury [18], while the correlation was non-significant at 10 years post injury [11]. Similarly, when the effect of sociodemographic factors, injury-related variables, cognitive appraisals, and coping strategies were controlled for, the association between acceptance and depression was inconsistent. Specifically, acceptance was a significant predictor of depression at 12 weeks and 1 year post injury, and at 1 month post discharge from rehabilitation [13, 45, 46] while the results were non-significant at 2, 10, and 21 years post injury $[11,18,65]$. Lastly, one study used latent growth mixture modeling to explore trajectories of depression and found individuals high in acceptance to show favorable trajectories of stable low depression and improvement when followed over a 2-year period [49].

\section{Anxiety}

Associations between acceptance and anxiety were explored in 18 studies $[11,13,18,25,33,37-40,45-$ $47,49,58,62-64,67]$. Five cross-sectional studies found that greater acceptance was related to lower anxiety $[13,25,38,40,62]$, while four reported nonsignificant results [37, 47, 63, 67]. When sociodemographic factors, injury-related variables, and coping strategies were controlled for, acceptance was a significant predictor of anxiety in four cross-sectional studies [13, 39, 40, 62], while two reported nonsignificant results [13, 63]. One longitudinal study conducted a series of regression analyses using cross-sectional data, which indicated that acceptance was only associated with anxiety in the early stages of an injury (i.e., at 6 and 12 weeks post injury), while it was not at 24 weeks post injury nor at 1 month and 1 year post discharge [13]. In the longitudinal studies, acceptance at six to 12 weeks post injury was significantly correlated with anxiety at 1 [45] and 2 years post injury [18], but not at 10 years [11]. When the effect of sociodemographic factors, injury-related variables, and coping strategies were controlled for, acceptance did not contribute significantly to anxiety scores at 12 weeks [46], 1 year [45], 2 years [18], or 10 years post injury [11], nor at 1 month post discharge from rehabilitation [13]. Thus, while acceptance was associated with anxiety when the two were measured simultaneously, it was not associated with future anxiety scores when potential confounders were controlled for. Lastly, individuals high in acceptance presented with a favorable trajectory of stable low anxiety as compared with delayed anxiety and anxiety improvement when followed over a 2-year period [49].

\section{Post-traumatic stress disorder}

Two cross-sectional studies explored the association between acceptance and PTSD [19, 51]. One found that greater acceptance correlated significantly with lower PTSD symptomology [19], while the other reported nonsignificant results [51]. The first study subdivided PTSD symptomatology into intrusion and avoidance and found that higher acceptance significantly predicted lower intrusion scores over and above what was predicted by sociodemographic factors, injury-related variables, coping strategies from the COPE inventory, and participant country (Great Britain, Germany, and Switzerland) [19]. The association between acceptance and avoidance was not statistically significant [19]. No longitudinal studies explored this association.

\section{Discussion}

The aim of this systematic review was to identify, critically appraise, and synthesize relevant research findings on how acceptance is associated with QOL and mental health outcomes in individuals living with SCI. Forty-one studies were included, which collectively showed that greater acceptance was related to favorable psychosocial outcomes. Specifically, greater acceptance was associated with greater global and psychological QOL, satisfaction with life, wellbeing, and with lower levels of depression and anxiety. Inconsistent evidence was found with regards to social QOL and PTSD. In the majority of studies, acceptance was a significant predictor of these adjustment outcomes even when sociodemographic factors, injury-related and psychosocial variables like social support, cognitive appraisals, and coping strategies were controlled for. This was an important finding as it indicated that the observed associations were not simply due to between-group differences such as injury severity. This systematic review also identified a range of methodological issues across studies, including reliance on cross-sectional designs, lack of statistical control for confounders, poorly defined study populations, and low participation rates.

\section{The declining direct influence of acceptance over time}

Acceptance, QOL, and mental health outcomes were much less consistently related in the longitudinal compared with 
the cross-sectional studies. Acceptance predicted favorable trajectories of depression and anxiety over a 2 year period [49], and further predicted scores of both QOL and depression up to 1 year following injury $[45,46]$. Conversely, acceptance did not predict QOL, depression, nor anxiety at 2,10 , or 21 years following injury $[11,18,65]$. Notably, these longitudinal studies struggled with small sample sizes (see Table 1), so it might be explained by a lack of statistical power [68]. However, it could also suggest that acceptance does not predict favorable long-term adjustment. In this light, acceptance may function primarily as a catalyst initiating favorable adjustment but declines in direct influence as the individual gradually adjusts to the new life circumstances. This could be interpreted in line with the SCIAM, which suggest that the adjustment process is dynamic and self-reinforcing (i.e., greater acceptance can lead to more favorable adjustment outcomes, and these in turn lead to even greater acceptance, and so on) [20]. Initial acceptance may, therefore, not predict future adjustment levels because mediational and self-reinforcing processes has occurred between the two time points. This interpretation finds some support in this review as the association between acceptance and later QOL and mental health disappeared only when additional psychosocial variables were included in regression models. This trend suggests that the direct effect of acceptance on later QOL and mental health declines over time, and either suggests that acceptance has little or no relevance over time, or that its long-term effect is mediated by other variables.

\section{Potential mediational effects}

Unfortunately, such mediational effects were not explicitly tested in the included studies. However, a potentially relevant mediator was positive reinterpretation from the COPE inventory, which refers to construing a stressful situation in positive terms [16]. While acceptance was a significant predictor of both depression and psychological QOL, this effect disappeared whenever positive reinterpretation was added to regression models (see Table 1). There are several possible explanations. First, it could simply suggest that the aspects of acceptance that significantly predict QOL and depression share variance with other relevant constructs. It could also suggest a mediational relationship in which greater initial acceptance leads to perceiving the injury in more positive terms, a frame of mind more directly associated with lower depression and greater psychological QOL.

\section{The value-change aspect of acceptance}

In the longitudinal studies, including the value-change aspect of acceptance (i.e., measuring acceptance with the
SCL-CSQ) generally led to significant results while omitting it (i.e., measuring acceptance with the COPE inventory) often led to nonsignificant results (see Table 1). In the transactional model of stress and coping, acceptance is assumed to decrease psychological distress by reducing the discrepancy between priorities in life and what one is capable of doing after SCI or other disability onset [25]. In this framework, it may, therefore, not be enough to accept the reality of the situation if it does not lead to a process of value-change. The results of this review tentatively supported this framework and highlighted the importance of the value-change aspect of acceptance when considering the temporal associations between acceptance, QOL, and mental health outcomes.

\section{Clinical implications}

Health-care professionals in rehabilitation today often, explicitly or implicitly, rely on the foundational principles of rehabilitation psychology where acceptance, as elaborated by Wright, is a core principle of adjustment [69]. This systematic review adds empirical weight to this perspective as the synthesis highlighted acceptance as a psychological resource supporting the adjustment process following lifechanging injuries. Individuals with greater acceptance of their new life circumstances may reduce psychological distress by reducing the discrepancy between priorities in life and what they are capable of doing. However, it is still unclear whether acceptance of SCI can be improved through psychotherapy, and whether an increase in acceptance would lead to favorable outcomes. This could be explored by investigating the effectiveness of therapeutic approaches that stimulate and support the acceptance process. Acceptance and Commitment Therapy (ACT) [70] might be promising as it aims to stimulate acceptance of what cannot be changed [70], and it has shown encouraging results for individuals with other severely disabling health conditions such as cancer [71] and multiple sclerosis [72].

\section{Methodological limitations of the included studies}

The main issues in study quality were low participation rates, cross-sectional study designs, poorly defined study samples, and a lack of statistical control for confounders (see Supplementary Appendix 2). The low participation rates and poorly defined study samples are a major issue because the findings might only be representative of a subset of individuals with SCI who are highly engaged in research. Furthermore, the widespread use of crosssectionally designed studies with correlational results is a major limitation because it does not permit temporal or causal inferences. Greater acceptance may thus follow from being less depressed and having greater QOL rather than the 
other way around. Longitudinal studies are important in teasing out these temporal effects [73], but unfortunately most of the longitudinal studies included were based on limited sample sizes.

Another concern was the exclusive use of self-report measures. In self-reporting it is unclear what the respondent is thinking whilst filling out the questionnaire. This is a concern because acceptance may imply resignation or giving up to some respondents. For example, a qualitative study in chronic pain patients found that acceptance was perceived by some participants as an empowerment process, while others thought of acceptance as a personal failure [74].

Lastly, mediational effects were not explored in the included studies. Investigating how acceptance is associated with QOL and mental health outcomes within a mediational framework, like the SCIAM, could have important theoretical and clinical implications.

\section{Limitations of this review}

One of the major limitations of this systematic review was the inclusion of studies with different measurement scales and methodologies such as cross sectional but also longitudinal data and bivariate but also multivariate data. This all-encompassing approach provided an overview of the research field, but was also a limitation, because many of the studies were not directly comparable. This complicated the data synthesis, and it further prohibited the use of meta-analysis to quantitatively synthesize data. Another limitation was the lack of explicit use of study quality in the selection of studies and data synthesis, where poorly designed studies with considerable risk of bias was not weighed differently than well-designed studies. A further limitation pertains to the lack of evaluation of potential reporting and publication biases. Reporting bias refers to selective reporting of results within studies [75], while publication bias refers to a bias in published academic research where positive and significant results are more likely to be published than negative or nonsignificant results [76]. These biases are well known in intervention studies, but it may also be a problem in observational studies where many different variables are examined and only some associations are fully analyzed, reported, and published [77]. However, no analysis of quality across studies was conducted, so the degree to which this has happened cannot be determined. Furthermore, acceptance might be associated in relevant ways to narrower constructs such as hopelessness, functional independence, or social isolation, but these were not included in this review. Lastly, only papers published in English, Danish, Norwegian, or Swedish were included, potentially excluding relevant studies published in another language.

\section{Future research}

Future studies should first of all prioritize addressing methodological issues identified in this review. For example, they should focus on conducting longitudinal studies with sufficiently large sample sizes to tease out the temporal relationships. Moreover, how individuals with SCI understand the construct of acceptance should be explored with methods such as the cognitive interviewing approach [78], which seems promising as it systematically explores whether participants attach the intended meaning to questionnaire items [78]. In addition, future studies should explore the associations between acceptance, QOL, and mental health outcomes within a dyadic perspective where the respondents and their partner are perceived as interdependent and mutually influence each other in their adjustment process [79]. Research from related fields of disability research have shown significant dyadic effects [80], but this approach was not reflected in any of the included studies, nor in the theoretical models. It might be valuable for future studies to investigate dyadic processes and potentially revise the theoretical models accordingly. Lastly, future studies should investigate the effectiveness of therapeutic approaches that stimulate and support the acceptance process, such as ACT [70].

\section{Conclusion}

Based on 41 studies, this systematic review highlighted the consistent association of acceptance with QOL and mental health following SCI. Generally, acceptance predicted favorable adjustment outcomes up to a 2-year period, while it did not predict adjustment outcomes at 10 or 21 years following injury. This systematic review also identified a range of methodological issues, including cross-sectional designs and lack of statistical control for confounders. Future studies should prioritize addressing these issues to enhance study quality, and it might be valuable to consider dyadic effects, explore the subjective meaning(s) of acceptance, and investigate the effectiveness of therapeutic approaches that stimulate and support the acceptance process.

Acknowledgements The authors thank the research librarians at University of Southern Denmark for providing feedback on the search algorithm.

Funding The present paper is part of a Ph.D. project funded by The Specialized Hospital for Polio and Accident Victims in Roedovre, Denmark.

Author contributions AA was responsible for designing and writing the review protocol, conducting the literature search, screening identified records, conducting quality assessment, extracting and synthesizing data, interpreting results, creating figures and tables, 
and writing the manuscript. SLR was responsible for screening identified records, conducting quality assessment, and extracting and synthesizing data. She further contributed with guidance and continuous feedback on the review protocol, tables and figures, and revisions of the manuscript. HK contributed with guidance and continuous feedback on the review protocol, tables and figures, and revisions of the manuscript. TEA was responsible for arbitrating potentially eligible studies and further contributed with guidance and continuous feedback on the review protocol, tables and figures, and revisions of the manuscript.

\section{Compliance with ethical standards}

Conflict of interest The authors declare that they have no conflict of interest.

Publisher's note Springer Nature remains neutral with regard to jurisdictional claims in published maps and institutional affiliations.

\section{References}

1. Crewe NM, Krause JS. Spinal cord injury. In: Brodwin MG, Siu FW, Howard J, Bronwin ER, editors. Medical, psychosocial and vocational aspects of disability. 3rd ed. Athens, GA: Elliott \& Fitzpatrick, Inc.; 2009. p. 289-305.

2. Heinemann AW, Wilson CS, Huston T, Koval J, Gordon S, Gassaway J, et al. Relationship of psychology inpatient rehabilitation services and patient characteristics to outcomes following spinal cord injury: the SCIRehab Project. J Spinal Cord Med. 2012;35:578-92. http://www.tandfonline.com/doi/full/10.1179/ 2045772312 Y.0000000059.

3. Phillips BN, Smedema SM, Fleming AR, Sung C, Allen MG. Mediators of disability and hope for people with spinal cord injury. Disabil Rehabil. 2016;38:1672-83. http://www.ta ndfonline.com/doi/full/10.3109/09638288.2015.1107639.

4. van Leeuwen C, Kraaijeveld S, Lindeman E, Post MWM. Associations between psychological factors and quality of life ratings in persons with spinal cord injury: a systematic review. Spinal Cord. 2012;50:174-87.

5. Ottomanelli L, Lind L. Review of critical factors related to employment after spinal cord injury: implications for research and vocational services. J Spinal Cord Med. 2009;32:503-31.

6. Kreuter M. Spinal cord injury and partner relationships. Spinal Cord. 2000;38:2-6.

7. Barclay L, McDonald R, Lentin P, Bourke-Taylor H. Facilitators and barriers to social and community participation following spinal cord injury. Aust Occup Ther J. 2016;63:19-28. http://doi. wiley.com/10.1111/1440-1630.12241.

8. Post MWM, van Leeuwen CMC. Psychosocial issues in spinal cord injury: a review. Spinal Cord. 2012;50:382-9. http://www.na ture.com/doifinder/10.1038/sc.2011.182.

9. Craig A, Perry KN, Guest R, Tran Y, Middleton J. Adjustment following chronic spinal cord injury: Determining factors that contribute to social participation. $\mathrm{Br} \mathrm{J}$ Health Psychol. 2015;20:807-23. http://doi.wiley.com/10.1111/bjhp.12143.

10. Migliorini CE, New PW, Tonge BJ. Comparison of depression, anxiety and stress in persons with traumatic and non-traumatic post-acute spinal cord injury. Spinal Cord. 2009;47:783. https:// doi.org/10.1038/sc.2009.43.

11. Pollard C, Kennedy P. A longitudinal analysis of emotional impact, coping strategies and post-traumatic psychological growth following spinal cord injury: a 10-year review. $\mathrm{Br} \mathrm{J}$ Health Psychol. 2007;12:347-62. http://doi.wiley.com/10.1348/ $135910707 X 197046$.
12. Elfström ML, Kreuter M, Persson L-O, Sullivan M. General and condition-specific measures of coping strategies in persons with spinal cord lesion. Psychol Health Med. 2005;10:231-42. http://www.tandfonline.com/doi/abs/10.1080/1354850041233133 4136.

13. Kennedy P, Marsh N, Lowe R, Grey N, Short E, Rogers B. A longitudinal analysis of psychological impact and coping strategies following spinal cord injury. $\mathrm{Br} \mathrm{J}$ Health Psychol. 2000;5:157-72.

14. Peter C, Müller R, Post MWM, van Leeuwen CMC, Werner CS, Geyh S. Psychological resources, appraisals, and coping and their relationship to participation in spinal cord injury: a path analysis. Arch Phys Med Rehabil. 2014;95:1662-71. http://linkinghub. elsevier.com/retrieve/pii/S0003999314003281.

15. Elfström ML, Rydén A, Kreuter M, Persson L-O, Sullivan M. Linkages between coping and psychological outcome in the spinal cord lesioned: development of SCL-related measures. Spinal Cord. 2002;40:23-9.

16. Carver CS, Scheier MF, Weintraub JK. Assessing coping strategies: a theoretically based approach. J Pers Soc Psychol. 1989;56:267-83. http://doi.apa.org/getdoi.cfm?doi=10.1037/0022-3514.56.2.267.

17. Elfström ML, Rydén A, Kreuter M, Taft C, Sullivan M. Relations between coping strategies and health-related quality of life in patients with spinal cord lesion. J Rehabil Med. 2005;37:9-16. https://medicaljournals.se/jrm/content/abstract/10.1080/16501970 410034414.

18. Kennedy P, Lude P, Elfström ML, Smithson E. Appraisals, coping and adjustment pre and post SCI rehabilitation: a 2-year follow-up study. Spinal Cord. 2012;50:112-8.

19. Lude P, Kennedy P, Evans M, Lude Y, Beedie A. Post traumatic distress symptoms following spinal cord injury: a comparative review of European Samples. Spinal Cord. 2005;43:102-8.

20. Craig A, Tran Y, Middleton J. Theory of adjustment following severe neurological injury: evidence supporting the spinal cord injury adjustment model. Horizons Neurosci Res. 2017;29: 117-139.

21. Cairns D, Baker J. Adjustment to spinal cord injury: a review of coping styles contributing to the process. J Rehabil. 1993;4:30-3.

22. Wright BA. Physical disability: a psychosocial approach. 2nd ed. New York: Harper \& Row; 1983.

23. Keany KC, Glueckauf RL. Disability and value change: an overview and reanalysis of acceptance of loss theory. Rehabil Psychol. 1993;38:199-210. http://doi.apa.org/getdoi.cfm?doi=10. 1037/h0080297.

24. Lazarus RS, Folkman S. Stress, appraisal, and coping. New York: Springer Publishing Company; 1984.

25. Elfström ML, Kennedy P, Lude P, Taylor N. Condition-related coping strategies in persons with spinal cord lesion: a crossnational validation of the Spinal Cord Lesion-related Coping Strategies Questionnaire in four community samples. Spinal Cord. 2007;45:420-8. http://www.nature.com/articles/3102003.

26. Moher D, Liberati A, Tetzlaff J, Altman DG, The PRISMA Group. Preferred reporting items for systematic reviews and metaanalyses: the PRISMA statement. PLOS Med. 2009;6. https://doi. org/10.1371/journal.pmed.1000097.

27. Linkowsky D. A scale to measure acceptance of disability. Rehabil Couns Bull. 1971;14:236-44.

28. WHOQOL Group. The World Health Organization Quality of Life Assessment (WHOQOL). Soc Sci Med. 1995;41:1403-9.

29. Kroenke K, Spitzer RL, Williams JBW. The PHQ-9: validity of a brief depression severity measure. $\mathrm{J}$ Gen Intern Med. 2001;16:606-13. http://link.springer.com/10.1046/j.1525-1497. 2001.016009606.x.

30. Veritas Health Innovation. Covidence systematic review software. Melbourne, Australia: Veritas Health Innovation. www.covidence. org. Accessed: August 30, 2018. 
31. National Institutes of Health. Study quality assessment tools. National Institutes of Health. https://www.nhlbi.nih.gov/healthtopics/study-quality-assessment-tools. Accessed: October 11, 2018.

32. Critical Appraisal Skills Programme. CASP Qualitative Checklist. www.casp-uk.net/casp-tools-checklists/2018. Accessed: October $11,2018$.

33. Migliorini CE, Elfström ML, Tonge BJ. Translation and Australian validation of the spinal cord lesion-related coping strategies and emotional wellbeing questionnaires. Spinal Cord. 2008;46: 690-5.

34. Migliorini CE, Tonge BJ. Reflecting on subjective well-being and spinal cord injury. J Rehabil Med. 2009;41:445-50.

35. Migliorini CE, New PW, Tonge BJ. Quality of life in adults with spinal cord injury living in the community. Spinal Cord. 2011;49:365-70. https://doi.org/10.1038/sc.2010.102.

36. Miller SM, Chan F, Ferrin JM, Lin C-P, Chan JYC. Confirmatory factor analysis of the World Health Organization Quality of life questionnaire-brief version for individuals with spinal cord injury. Rehabil Couns Bull. 2008;51:221-8. http://journals.sa gepub.com/doi/10.1177/0034355208316806.

37. Perry KN, Nicholas MK, Middleton J, Siddall P. Psychological characteristics of people with spinal cord injury-related persisting pain referred to a tertiary pain management center. J Rehabil Res Dev. 2009;46:57. http://www.rehab.research.va.gov/jour/09/46/1/ perry.html.

38. Perry KN, Nicholas MK, Middleton J. Spinal cord injury-related pain in rehabilitation: a cross-sectional study of relationships with cognitions, mood and physical function. Eur $\mathrm{J}$ Pain. 2009;13:511-7. https://doi.org/10.1016/j.ejpain.2008.06.003.

39. Saffari M, Pakpour AH, Yaghobidoot M, Al Zaben F, Koenige HG. Cross-cultural adaptation of the spinal cord lesion-related coping strategies questionnaire for use in Iran. Injury. 2015;46:1539-44. https://doi.org/10.1016/j.injury.2015.04.035.

40. Saurí J, Umaña MC, Chamarro A, Soler MD, Gilabert A, Elfström ML. Adaptation and validation of the Spanish version of the Spinal Cord Lesion-related Coping Strategies Questionnaire (SCL CSQ-S). Spinal Cord. 2014;52:842-9. http://www.nature.com/a rticles/sc201444.

41. Wollaars MM, Post MWM, Asbeck FWAVan, Brand N. Spinal Cord Injury pain: the influence of psychologic factors and impact on quality of life. Clin J Pain. 2007;23:383-91.

42. Elfström ML, Kreuter M. Relationships between locus of control, coping strategies and emotional well-being in persons with Spinal Cord Lesion. J Clin Psychol Med Settings. 2006;13:89-100. http://link.springer.com/10.1007/s10880-005-9001-8.

43. Ferdiana A, Post MWM, King N, Bültmann U, van der Klink JJL. Meaning and components of quality of life among individuals with spinal cord injury in Yogyakarta Province, Indonesia. Disabil Rehabil. 2018;40:1183-91. https://doi.org/10.1080/09638288. 2017.1294204.

44. Ferrin JM, Chan Fong, Chronister J, et al. Psychometric validation of the multidimensional acceptance of loss scale. Clin Rehabil. 2011;25:166-74. http://journals.sagepub.com/doi/10. 1177/0269215510380836.

45. Kennedy P, Lude P, Elfström ML, Smithson E. Sense of coherence and psychological outcomes in people with spinal cord injury: appraisals and behavioural responses. Br J Health Psychol. 2010;15:611-21.

46. Kennedy P, Lude P, Elfström ML, Smithson E. Cognitive appraisals, coping and quality of life outcomes: A multi-centre study of spinal cord injury rehabilitation. Spinal Cord. 2010;48: 762-9.

47. Kennedy P, Nolan M, Smithson E. Psychological adjustment to spinal cord injury in Ireland: quality of life, appraisals and coping. Ir J Psychol. 2011;32:116-29.
48. Khodayarian M, Tafti BF, Zare A, Maghsoudi Z. Relationship between coping strategies and quality of life of persons with spinal cord injury: a cross-sectional study in Iran. Int $\mathbf{J}$ Adv Biotechnol Res. 2017;8:1545-56.

49. Bonanno GA, Kennedy P, Galatzer-Levy IR, Lude P, Elfström ML. Trajectories of resilience, depression, and anxiety following spinal cord injury. Rehabil Psychol. 2012;57:236-47. http://doi. apa.org/getdoi.cfm?doi=10.1037/a0029256.

50. Smedema SM, Catalano D, Ebener DJ. The relationship of coping, self-worth, and subjective well-being: a structural equation model. Rehabil Couns Bull. 2010;53:131-42. http://journals.sagepub. com/doi/10.1177/0034355209358272.

51. DeRoon-Cassini TA, De St. Aubin E, Valvano AK, Hastings J, Brasel KJ. Meaning-making appraisals relevant to adjustment for veterans with spinal cord injury. Psychol Serv 2013;10:186-93.

52. Catalano D, Kim J-H, Ditchman NM, Shin H-U, Lee J, Chan F. The sense of well-being inventory as a quality of life measure for people with spinal cord injury. Aust $\mathrm{J}$ Rehabil Couns. 2010;16:57-72. https://www.cambridge.org/core/product/identifier/ S1323892200020524/type/journal_article.

53. Anderson CJ, Vogel LC, Chlan KM, Betz RR. Coping with spinal cord injury: strategies used by adults who sustained their injuries as children or adolescents. J Spinal Cord Med. 2008;31:290-6.

54. Smedema SM. Evaluation of a concentric biopsychosocial model of well-being in persons with spinal cord injuries. Rehabil Psychol. 2017;62:186-97. http://doi.apa.org/getdoi.cfm?doi=10. 1037/rep0000150.

55. Leeuwen C, Edelaar-Peeters Y, Peter C, Stiggelbout A, Post M. Psychological factors and mental health in persons with spinal cord injury: An exploration of change or stability. J Rehabil Med. 2015;47:531-7. http://www.medicaljournals.se/jrm/content/?doi= $10.2340 / 16501977-1953$.

56. van Leeuwen C, Edelaar-Peeters Y, Peter C, Stiggelbout A, Post M. Psychological factors and mental health in persons with spinal cord injury: an exploration of change or stability. J Rehabil Med. 2015;47:531-7. http://www.medicaljournals.se/jrm/content/?doi= 10.2340/16501977-1953.

57. Berry JW, Elliott TR, Rivera P. Resilient, undercontrolled, and overcontrolled personality prototypes among persons with spinal cord injury. J Pers Assess. 2007;89:292-302. http://www.ta ndfonline.com/doi/abs/10.1080/00223890701629813.

58. Carvalho SAD, de, Andrade MJ, Tavares MA, De Freitas JLS. Spinal cord injury and psychological response. Gen Hosp Psychiatry. 1998;20:353-9.

59. Elliott TR, Uswatte G, Lewis L, Palmatier A. Goal instability and adjustment to physical disability. J Couns Psychol. 2000;47:251-65. http://doi.apa.org/getdoi.cfm?doi=10.1037/0022-0167.47.2.251.

60. Jiao J, Heyne MM, Lam CS. Acceptance of disability among chinese individuals with spinal cord injuries: the effects of social support and depression. Psychology. 2012;3:775-81.

61. Jörgensen S, Ginis K, Iwarsson S, Lexell J. Depressive symptoms among older adults with long-term spinal cord injury: associations with secondary health conditions, sense of coherence, coping strategies and physical activity. J Rehabil Med. 2017;49:644-51. https://www.medicaljournals.se/jrm/content/abstract/10.2340/ 16501977-2259.

62. Kennedy P, Lowe R, Grey N, Short E. Traumatic spinal cord injury and psychological impact: a cross-sectional analysis of coping strategies. Br J Clin Psychol. 1995;34:627-39.

63. Kennedy P, Evans M, Sandhu N. Psychological adjustment to spinal cord injury: the contribution of coping, hope and cognitive appraisals. Psychol Health Med. 2009;14:17-33. http://www.ta ndfonline.com/doi/full/10.1080/13548500802001801.

64. Kennedy P, Lude P, Elfström ML, Smithson E. Psychological contributions to functional independence: a longitudinal investigation of spinal cord injury rehabilitation. Arch Phys Med 
Rehabil. 2011;92:597-602. https://doi.org/10.1016/j.apmr.2010. 11.016.

65. Kennedy P, Kilvert A, Hasson L. A 21-year longitudinal analysis of impact, coping, and appraisals following spinal cord injury. Rehabil Psychol. 2016;61:92-101. http://doi.apa.org/getdoi.cfm? doi $=10.1037 /$ rep0000066.

66. Nicholls E, Lehan T, Plaza SLO, Deng X, Romero JLP, Pizarro JAA, et al. Factors influencing acceptance of disability in individuals with spinal cord injury in Neiva, Colombia, South America. Disabil Rehabil. 2012;34:1082-8.

67. Paker N, Bugdayci D, Kesiktas N, Sahin M, Elfström ML. Reliability and validity of the Turkish version of spinal cord lesion-related coping strategies. Spinal Cord. 2014;52:383-7.

68. Field A. Discovering statistics using IBM SPSS statistics. 4th ed. London: Sage Publications; 2013.

69. Dunn DS, Ehde DM, Wegener ST. The foundational principles as psychological lodestars: Theoretical inspiration and empirical direction in rehabilitation psychology. Rehabil Psychol. 2016;61:1-6.

70. Hayes SC, Strosahl KD, Wilson KG. Acceptance and commitment therapy: the process and practice of mindful change. 2nd ed. New York: Guilford Press; 2012.

71. Fashler SR, Weinrib AZ, Azam MA, Katz J. The use of acceptance and commitment therapy in oncology settings: a narrative review. Psychol Rep. 2017;121:229-52. https://doi.org/10.1177/ 0033294117726061.

72. Nordin L, Rorsman I. Cognitive behavioural therapy in multiple sclerosis: a randomized controlled pilot study of acceptance and commitment therapy. J Rehabil Med. 2012;44:87-90. http://www. medicaljournals.se/jrm/content/?doi=10.2340/16501977-0898.
73. Menard S. Handbook of longitudinal research: design, measurements and analysis. San Diego, CA: Academic Press; 2007.

74. Biguet G, Nilsson Wikmar L, Bullington J, Flink B, Löfgren M. Meanings of "acceptance" for patients with long-term pain when starting rehabilitation. Disabil Rehabil. 2016;38:1257-67. http://www.tandfonline.com/doi/full/10.3109/09638288.2015. 1076529.

75. Norris SL, Holmer HK, Ogden LA, Fu R, Abou-Setta AM, Viswanathan MS, McPheeters ML. Selective outcome reporting as a source of bias in reviews of comparative effectiveness. Rockville, MD: Agency for Healthcare Research and Quality. August 2012.

76. Song F, Parekh S, Hooper L, Loke Y, Ryder J, Sutton A, et al. Dissemination and publication of research findings: an updated review of related biases. Health Technol Assess (Rockv). 2010;14:1-220. https://www.journalslibrary.nihr.ac.uk/hta/hta14080/.

77. Dekkers OM, Vandenbroucke JP, Cevallos M, Renehan AG, Altman DG, Egger M. COSMOS-E: Guidance on conducting systematic reviews and meta-analyses of observational studies of etiology. PLoS Med. 2019;16:e1002742-e1002742. https://www. ncbi.nlm.nih.gov/pubmed/30789892.

78. Hak T, van der Veer K, Jansen H. The three-step test-interview (TSTI): an observation-based method for pretesting selfcompletion questionnaires. Surv Res Methods. 2008;2:143-50.

79. Bodenmann G. A systemic-transactional conceptualization of stress and coping in couples. Swiss J Psychol. 1995;54:34-49.

80. Pakenham KI, Samios C. Couples coping with multiple sclerosis: a dyadic perspective on the roles of mindfulness and acceptance. $\mathrm{J}$ Behav Med. 2013;36:389-400. http://link.springer.com/10.1007/ s10865-012-9434-0. 